\title{
COMPLEMENTARITIES AND THE EXISTENCE OF STRONG BERGE EQUILIBRIUM
}

\author{
KeRIM KESKIN $^{1}$ AND H. ÇAĞRI SAĞLAM ${ }^{1}$
}

\begin{abstract}
This paper studies the existence and the order structure of strong Berge equilibrium, a refinement of Nash equilibrium, for games with strategic complementarities à la strong Berge. It is shown that the equilibrium set is a nonempty complete lattice. Moreover, we provide a monotone comparative statics result such that the greatest and the lowest equilibria are increasing.
\end{abstract}

Keywords. Strong Berge equilibrium, refinement, games with strategic complementarities, fixed point theory, supermodularity.

Mathematics Subject Classification. 91B52, 47H10.

\section{INTRODUCTION}

In this paper, we analyze the existence and the order structure of strong Berge equilibrium (hereafter, SBE) [1] in non-cooperative games with strategic complementarities.

SBE induces a refinement of Nash equilibrium [2] as it is immune not only to unilateral deviations, but also to the deviations of certain coalitions. According to the definition, only the complementary coalitions - defined for each player as the set of remaining players - are essential. This is where SBE differs from strong Nash equilibrium [3]; a well-known equilibrium notion satisfying coalitional stability. In addition, SBE requires the members of each complementary coalition to select a strategy profile that maximizes every members' well-being at the same time. This ensures a stronger stability than that of strong Nash equilibrium, but does not necessarily imply Pareto optimality since SBE does not consider the grand

Received May 31, 2013. Accepted January 20, 2014.

1 Department of Economics, Bilkent University, 06800 Ankara, Turkey.

kerim@bilkent.edu.tr 
coalition. Indeed, any SBE which is Pareto efficient is also a strong Nash equilibrium (see [4]). It is also clear that in a two-person non-cooperative game, SBE is equivalent to Nash equilibrium.

Earlier contributions on the existence of SBE are based on topologically oriented approaches that generally require convexity and compactness of strategy sets, and quasi-concavity of each payoff function in own action (see [4-7]). We instead resort to games with strategic complementarities (GSC) in which the strategy space is a complete lattice and the joint best response correspondence ${ }^{2}$ is Veinott-increasing. The class of GSC is arguably more convenient for studying SBE for the following reasons: (i) emphasizing the coalitions of players, SBE induces more coordination than pure competition; and (ii) SBE has much to offer as a refinement when players' potential to influence others' payoffs is substantial.

Introducing the order and the monotonicity properties of GSC à la strong Berge, we prove that the set of SBE is nonempty, and is indeed a complete lattice. Additional to the existence result which utilizes Zhou's [8] fixed point theorem, we refer to a constructive proof of Echenique [9] which indicates a method to compute the extremal equilibria, i.e., the greatest SBE and the least SBE. Moreover, based on Topkis [10], we provide a monotone comparative statics result on the equilibrium set such that for a collection of games, the extremal equilibria are increasing.

This paper is structured as follows: In Section 2, we give the definitions and theorems that are used throughout this study. Section 3 includes the results on the set of strong Berge equilibrium. Section 4 concludes.

\section{Definitions}

We call a partially ordered set lattice, if it contains the supremum and the infimum of all pairs of its elements. A lattice is complete if each nonempty subset has a supremum and an infimum. A subset $Y$ of a lattice $X$ is a subcomplete sublattice of $X$ if for each nonempty subset $Y^{\prime}$ of $Y, \bigvee_{X} Y^{\prime}$ and $\bigwedge_{X} Y^{\prime}$ exist and are contained in $Y$.

Let $X$ be a lattice. A function $f: X \rightarrow \mathbb{R}$ is supermodular in $x$ if $\forall x, x^{\prime} \in X$ :

$$
f(x)+f\left(x^{\prime}\right) \leq f\left(x \wedge x^{\prime}\right)+f\left(x \vee x^{\prime}\right) .
$$

Moreover, a function $f$ defined on $X \times T$ has increasing differences in $(x, t)$ if $f\left(x, t^{\prime}\right)-f(x, t)$ is increasing in $x$ for every $t<t^{\prime}$. A correspondence $F: X \rightarrow X$ is Veinott-increasing if for each $x, y \in X$ with $x<y, a \in F(x)$ and $b \in F(y)$ implies $a \wedge b \in F(x)$ and $a \vee b \in F(y)$. Finally, $x \in X$ is a fixed point of a correspondence $F: X \rightarrow X$, if $x \in F(x)$.

In addition, below are the theorems we utilize throughout this paper:

Theorem 2.1 Zhou [8]. Let $X$ be a nonempty complete lattice, and $F: X \rightarrow X$ be a nonempty-valued correspondence. If $F$ is Veinott-increasing and $F(x)$ is a

\footnotetext{
${ }^{2}$ In this paper, best response correspondences are according to SBE unless otherwise stated.
} 
subcomplete sublattice of $X$ for every $x \in X$, then the fixed point set of $F$ is a nonempty complete lattice.

Theorem 2.2 Topkis [10]. Let $X$ be a nonempty complete lattice, $T$ be a partially ordered set, and $Y: X \times T \rightarrow X \times T$ be a correspondence. If $Y$ is Veinott-increasing and $Y(x, t)$ is a nonempty subcomplete sublattice of $X \times T$ for each $(x, t)$, then

(i) $\forall t \in T$, there exists a greatest (least) fixed point of $Y(x, t)$;

(ii) the greatest (least) fixed point of $Y(x, t)$ is increasing in $t$ on $T$.

We define the notion of SBE as follows. Let $\Gamma=\left(N,\left(X_{i}\right)_{i \in N},\left(u_{i}\right)_{i \in N}\right)$ where $N$ is the player set, $X_{i}$ is the strategy set for agent $i$, and $u_{i}: X \rightarrow \mathbb{R}$ is the utility function of agent $i$.

Definition 2.3. In a game $\Gamma$, a strategy profile $x \in X$ is a strong Berge equilibrium (SBE) if $\forall i \in N, \forall j \in N \backslash\{i\}: u_{j}(x) \geq u_{j}\left(x_{i}, y_{-i}\right)$ for every $y_{-i} \in X_{-i}$.

Finally, for every $i \in N$, the coalitional best response correspondence $B_{-i}$ : $X \rightarrow X$ of the complementary coalition $-i$ is defined as follows:

$$
B_{-i}(x)=\left\{y \in X \mid \forall j \in N \backslash\{i\}, \forall y^{\prime} \in X: u_{j}\left(x_{i}, y_{-i}\right) \geq u_{j}\left(x_{i}, y_{-i}^{\prime}\right)\right\} .
$$

We follow the definition of Deghdak and Florenzano [7] for the joint best response correspondence, $B: X \rightarrow X$ :

$$
B(x)=\bigcap_{i \in N} B_{-i}(x)
$$

\section{ON THE SET OF STRONG BERGE EQUILIBRIUM}

In this section, we study a certain class of games, namely games with strategic complementarities (GSC) à la strong Berge. Notice that the following definition is similar to GSC à la Nash (see [11]), ${ }^{3}$ but is essentially different since corresponding best response correspondences are different.

Definition 3.1. A game has strategic complementarities à la strong Berge (or, is a GSC à la strong Berge) if (i) $\forall i \in N, X_{i}$ is a nonempty complete lattice; (ii) $B$ is nonempty-valued and Veinott-increasing in $x$ on $X$; and (iii) $\forall x \in X ; B(x)$ is a subcomplete sublattice of $X$.

A curious question that arises here is whether a GSC à la strong Berge is also a GSC à la Nash. Although this seems to be a valid statement which can follow from the fact that SBE is a refinement of Nash equilibrium, it turns out that one set does not contain the other. This fact will also lead to an interpretation that our existence result implies the existence of Nash equilibrium outside GSC à la Nash.

\footnotetext{
${ }^{3}$ In fact, these are called GSC in the literature, but we prefer "GSC à la Nash" to avoid confusion.
} 
Remark 3.2. There exist games $\Gamma$ and $\Gamma^{\prime}$, where $\Gamma$ is a GSC à la Nash but $\Gamma^{\prime}$ is not, and $\Gamma$ is not a GSC à la strong Berge but $\Gamma^{\prime}$ is.

Proof. First note that $B_{-i}$ and $B$ denote the coalitional best response correspondence and the joint best response correspondence, respectively. Moreover, let $B_{i}$ and $B_{N}$ denote the individual best response correspondence according to Nash equilibrium and the joint best response correspondence according to Nash equilibrium, respectively. Let $\Gamma$ be a three-player game which can be represented by the following game matrix.

$x_{3}$

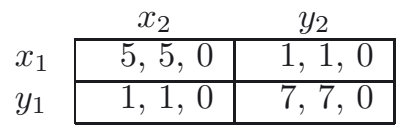

$y_{3}$

\begin{tabular}{l|r|r|}
\multicolumn{1}{c}{} & \multicolumn{1}{c}{$x_{2}$} & \multicolumn{1}{c}{$y_{2}$} \\
\cline { 2 - 3 }$x_{1}$ & $7,7,0$ & $1,1,0$ \\
\cline { 2 - 3 }$y_{1}$ & $1,1,0$ & $5,5,0$ \\
\cline { 2 - 3 } & &
\end{tabular}

For this game, $B_{1}\left(\cdot, x_{2}, x_{3}\right)=\left(x_{1}, \cdot, \cdot\right), B_{1}\left(\cdot, y_{2}, x_{3}\right)=\left(y_{1}, \cdot, \cdot\right), B_{1}\left(\cdot, x_{2}, y_{3}\right)=$ $\left(x_{1}, \cdot, \cdot\right), B_{1}\left(\cdot, y_{2}, y_{3}\right)=\left(y_{1}, \cdot, \cdot\right), B_{2}\left(x_{1}, \cdot, x_{3}\right)=\left(\cdot, x_{2}, \cdot\right), B_{2}\left(y_{1}, \cdot, x_{3}\right)=\left(\cdot, y_{2}, \cdot\right)$, $B_{2}\left(x_{1}, \cdot, y_{3}\right)=\left(\cdot, x_{2}, \cdot\right)$ and $B_{2}\left(y_{1}, \cdot, y_{3}\right)=\left(\cdot, y_{2}, \cdot\right)$. Moreover, $B_{3}$ is obvious. Letting $x_{i}<y_{i}$ for every $i \in N$, every $B_{i}$ is Veinott-increasing, and so is $B_{N}$. However, $B\left(x_{1}, y_{2}, x_{3}\right)=\varnothing$. Then, $\Gamma$ is a GSC à la Nash, but not a GSC à la strong Berge.

For $\Gamma^{\prime}$, we preserve the above notation and order, and represent the game as below:

$x_{3}$

\begin{tabular}{lr|r|}
\multicolumn{1}{c}{} & \multicolumn{1}{c}{$x_{2}$} & \multicolumn{1}{c}{$y_{2}$} \\
\cline { 2 - 3 }$x_{1}$ & $2,1,2$ & $0,0,0$ \\
\cline { 2 - 3 }$y_{1}$ & $2,2,2$ & $1,1,2$ \\
\cline { 2 - 3 } & &
\end{tabular}

$y_{3}$

\begin{tabular}{lr|r|}
\multicolumn{1}{c}{} & \multicolumn{1}{c}{$x_{2}$} & \multicolumn{1}{c}{$y_{2}$} \\
\cline { 2 - 3 }$x_{1}$ & $2,1,0$ & $0,0,1$ \\
\cline { 2 - 3 }$y_{1}$ & $2,2,1$ & $1,1,2$ \\
\cline { 2 - 3 } & &
\end{tabular}

The joint best response correspondence $B^{\prime}$ is nonempty-valued and Veinottincreasing since $B^{\prime}(\cdot, \cdot, \cdot)=\left(y_{1}, x_{2}, x_{3}\right)$ for every strategy profile. Moreover, $B_{3}^{\prime}\left(x_{1}, y_{2}, \cdot\right)=\left(\cdot, \cdot, y_{3}\right)$ and $B_{3}^{\prime}\left(y_{1}, y_{2}, \cdot\right)=(\cdot, \cdot, \cdot)$ which violates Veinottincreasingness of $B_{N}^{\prime}$. Then, although $\Gamma^{\prime}$ is a GSC à la strong Berge, it is not a GSC à la Nash.

In fact, the game $\Gamma^{\prime}$ of the above proof is a good example that shows how strong Berge equilibrium refines the set of Nash equilibrium by considering coalitional stability. It is quite easy to verify that the game has two Nash equilibria, $\left(x_{1}, x_{2}, x_{3}\right)$ and $\left(y_{1}, x_{2}, x_{3}\right)$, one of which is Pareto dominated by the other. Among these profiles, $\left(y_{1}, x_{2}, x_{3}\right)$ is the one which is coalitionally stable according to strong Berge equilibrium. The reason is that the coalition $\{1,2\}$ prefers $\left(y_{1}, x_{2}\right)$ when the strategy of agent 3 is fixed to $x_{3}$. Hence, the Pareto dominated Nash equilibrium gets eliminated. On the other hand, for this game, strong Nash equilibrium does not have much to offer as a refinement, since the set of strong Nash equilibirum coincides with the set of Nash equilibrium. For a more striking example to compare strong Berge equilibirum and strong Nash equilibirum, consider the famous 
Prisoner's Dilemma in which the set of strong Nash equilibrium turns out to be empty whereas strong Berge equilibrium can still make predictions.

We now present the existence theorem for SBE.

Theorem 3.3. A GSC à la strong Berge has a SBE. In fact, the equilibrium set is a complete lattice.

Proof. Noting that the set of fixed points of the joint best response correspondence is the set of SBE, the result trivially follows from Zhou's [8] theorem.

There are two important results which follow from Theorem 3.3. The former indicates that we show the existence of Nash equilibrium for GSC à la strong Berge which are not necessarily GSC à la Nash. The latter remark utilizes Echenique's [9] constructive proof, and highlights an approach to obtain the least SBE and the greatest SBE.

Remark 3.4. Nash equilibrium exists for GSC à la strong Berge.

Remark 3.5. The constructive proof of Echenique [9] directly applies here. Using the approach in the proof, the least equilibrium and the greatest equilibrium can be computed.

In the literature (see $[11,12]$ among others), sufficient conditions for games to be GSC à la Nash are provided: For each $i \in N$, if the utility function $u_{i}$ is supermodular in own strategies $x_{i}$ and has increasing differences in $\left(x_{i}, x_{-i}\right)$, then the conditions on the best response correspondences are satisfied. As a matter of fact, since utility functions are already defined within the game form, providing sufficient conditions on utility functions are considered to be more convenient. In this sense, the existence result can also be stated with conditions on utility functions.

For the following theorem, we borrow an assumption from Larbani and Nessah [5] to ensure the nonemptiness of the best response correspondences. Another assumption which serves this purpose would be to directly assume the nonempty-valuedness of the joint best response correspondence as it is done by Deghdak and Florenzano [7].

Theorem 3.6. In a game $\Gamma$, let each $X_{i}$ be a complete lattice. For every $i \in N$ and for every $j \in N \backslash\{i\}$, let $u_{i}$ be supermodular in $x_{-j}$ and have increasing differences in $\left(x_{j}, x_{-j}\right)$. Also assume that $\forall x \in X, \exists y \in X$ such that $\forall i \in N$, $\forall j \in N \backslash\{i\}, \forall z_{-i} \in X_{-i}$ :

$$
u_{i}\left(x_{i}, z_{-i}\right) \leq u_{i}\left(x_{i}, y_{-i}\right)
$$

Then the conditions of Definition 3.1 are satisfied. 
Proof. Since $\forall x \in X, \exists y \in X$ such that $\forall i \in N, \forall j \in N \backslash\{i\}, \forall z_{-i} \in X_{-i}$ : $u_{i}\left(x_{i}, z_{-i}\right) \leq u_{i}\left(x_{i}, y_{-i}\right)$ by assumption, the joint bet response correspondence is nonempty-valued.

For Veinott-increasingness, we need to show: for each $x, y \in X$ with $x<y$, if $a \in B(x)$ and $b \in B(y)$ then $a \wedge b \in B(x)$ and $a \vee b \in B(y)$. Take any $x, y \in X$ such that $x<y$. Then, pick any $a \in B(x)$ and $b \in B(y)$. By definition, $\forall i \in N$ : $a \in B_{-i}(x)$ and $b \in B_{-i}(y)$. Assume that $a \leq b$. We are trivially done. Now assume otherwise. We either have $a>b$ or we cannot compare $a$ and $b$. In either case, for a given $i \in N$, we have $\forall j \in N \backslash\{i\}$ :

$$
\begin{aligned}
0 \leq u_{j}\left(x_{i}, a_{-i}\right)-u_{j}\left(x_{i}, a_{-i} \wedge b_{-i}\right) & \leq u_{j}\left(x_{i}, a_{-i} \vee b_{-i}\right)-u_{j}\left(x_{i}, b_{-i}\right) \\
& \leq u_{j}\left(y_{i}, a_{-i} \vee b_{-i}\right)-u_{j}\left(y_{i}, b_{-i}\right) \leq 0 .
\end{aligned}
$$

Here, the first and the last inequalities are implied by optimality. The second inequality follows from supermodularity and the third inequality follows from increasing differences. It is obvious that each term is 0 which means $u_{j}\left(x_{i}, a_{-i}\right)=$ $u_{j}\left(x_{i}, a_{-i} \wedge b_{-i}\right)$ and $u_{j}\left(y_{i}, a_{-i} \vee b_{-i}\right)=u_{j}\left(y_{i}, b_{-i}\right)$. That is $a \wedge b \in B_{-i}(x)$ and $a \vee b \in B_{-i}(y)$. Since $i$ is arbitrary, $a \wedge b \in B(x)$ and $a \vee b \in B(y)$. Thus, $B$ is Veinott-increasing.

To show that $B(x)$ is a subcomplete sublattice, take any $x \in X$ and any $a, b \in$ $B(x)$. Consider any $i \in N$. Note that $a, b \in B_{-i}(x)$ as well. Then, by definition, $\forall j \in N \backslash\{i\}:$

$$
u_{j}\left(x_{i}, a_{-i}\right)=u_{j}\left(x_{i}, b_{-i}\right) \geq u_{j}\left(x_{i}, x_{-i}^{\prime}\right) \quad \forall x_{-i}^{\prime} \in X_{-i} .
$$

We now have $u_{j}\left(x_{i}, a_{-i}\right)+u_{j}\left(x_{i}, b_{-i}\right) \leq u_{j}\left(x_{i}, a_{-i} \wedge b_{-i}\right)+u_{j}\left(x_{i}, a_{-i} \vee b_{-i}\right)$ by supermodularity. And because of the optimality of $a$ and $b$, it can be concluded that $a \wedge b$ and $a \vee b$ are also in $B_{-i}(x)$. Since $i$ is arbitrary, $a \wedge b$ and $a \vee b$ are in $B(x)$ as well. Thus, for every $x \in X, B(x)$ is a subcomplete sublattice of $X$.

The normal form game $\Gamma^{\prime \prime}$ represented by the following game matrix satisfies the conditions given in Thereom 4 . For this game, the unique strong Berge equilibrium is $\left(y_{1}, x_{2}, y_{3}\right)$, whereas $\left\{\left(x_{1}, x_{2}, x_{3}\right),\left(x_{1}, x_{2}, y_{3}\right),\left(y_{1}, x_{2}, y_{3}\right),\left(y_{1}, y_{2}, y_{3}\right)\right\}$ is the set of Nash equilibria. Note that these strategy profiles are also strong Nash equilibria.

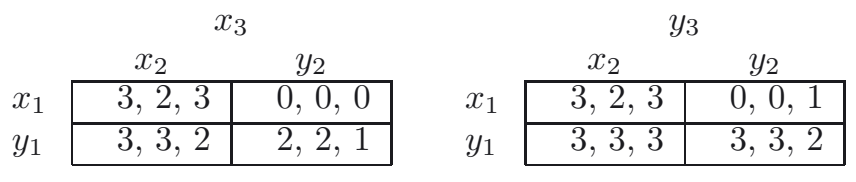

Finally, the following theorem provides a comparative statics result for the equilibrium set. This result is important for us to better understand the characteristics of SBE. For instance, the theorem indicates how SBE changes in a certain parameter for a collection of games. 
Theorem 3.7. Let $T$ be a partially ordered set, and $\left(\Gamma^{t}\right)_{t \in T}$ be a collection of $G S C$ à la strong Berge. Define $\mathcal{B}: X \times T \rightarrow X \times T$ to be the joint best response correspondence in $\Gamma^{t}$ such that for every $(x, t), \mathcal{B}(x, t)$ is the set of all $(y, t)$ where $y \in B(x)$. If $\mathcal{B}$ is Veinott increasing in $(x, t)$ on $X \times T$ and $\mathcal{B}(x, t)$ is a subcomplete sublattice of $X \times T$ for every $(x, t)$, then the extremal $S B E$ are increasing in $t$ on $T$.

Proof. Utilizing Topkis's [10] theorem, one can conclude that the greatest (least) fixed point of $\mathcal{B}$ is increasing in $t$ on $T$. Thus, the greatest $\mathrm{SBE}$ and the least $\mathrm{SBE}$ are both increasing in $t$ on $T$.

Consider a collection of GSC à la strong Berge characterized by normal form games $\Gamma^{\prime}$ and $\Gamma^{\prime \prime}$ we have analyzed above. Let $T=\{1,2\}, \Gamma^{1}=\Gamma^{\prime}$ and $\Gamma^{2}=\Gamma^{\prime \prime}$. This collection satisfies the conditions of the theorem, therefore we can conclude (even without analyzing the sets of equilibrium) that the extremal equilibria are increasing in $t$.

\section{Concluding Remarks}

In this paper, we prove the existence of SBE for GSC à la strong Berge. Our results differ from the existence results available in the current literature in the sense that we do not require convex and compact strategy sets, or any type of continuity for utility functions. Moreover, we provide a monotone comparative statics result on the equilibrium set such that for a collection of games, the extremal equilibria are increasing.

\section{REFERENCES}

[1] C. Berge, Théorie Générale des Jeux à n Personnes, Gautier Villars, Paris (1957).

[2] J.F. Nash, Non-cooperative games. Annal. Math. 54 (1951) 286-295.

[3] R. Aumann, Acceptable points in a general cooperative n-person games. in Contributions to the Theory of Games IV. Annal. Math. Study 40 (1959) 287-324.

[4] R. Nessah, M. Larbani and T. Tazdaï, Strong Berge equilibrium and strong Nash equilibrium: Their relation and existence, in Game Theory Appl., edited by L.A. Petrosjan and V.V. Mazalov. Vol. 15. Nova Science Publishers (2012) 165-180.

[5] M. Larbani and R. Nessah, Sur l'équilibre fort selon Berge. RAIRO Oper. Res. 35 (2001) 439-451.

[6] K.Y. Abalo and M.M. Kostreva, Intersection theorems and their applications to Berge equilibria. Appl. Math. Comput. 182 (2006) 1840-1848.

[7] M. Deghdak and M. Florenzano, On the existence of Berge's strong equilibrium. Int. Game Theory Rev. 13 (2011) 325-340.

[8] L. Zhou, The set of Nash equilibria of a supermodular game is a complete lattice. Games Econ. Behavior 7 (1994) 295-300.

[9] F. Echenique, A short and constructive proof of Tarski's fixed-point theorem. Int. J. Game Theory 33 (2005) 215-218.

[10] D.M. Topkis, Supermodularity and Complementarity, Princeton University Press, Princeton (1998).

[11] X. Vives, Complementarities and games: New developments. J. Econ. Literature 43 (2005) 437-479.

[12] R.W. Cooper, Coordination Games: Complementarities and Macroeconomics, Cambridge University Press, Cambridge (1999). 